\title{
What are we doing when we double check?
}

Yvonne Pfeiffer (DD , ${ }^{1}$ Chantal Zimmermann, ${ }^{1}$ David L B Schwappach (1) ${ }^{1,2}$

- Additional material is published online only. To view please visit the journal online (http://dx.doi.org/10.1136/ bmjqs-2019-009680).

${ }^{1}$ Research Department, Patient Safety Foundation, Zurich, Switzerland

${ }^{2}$ Institute of Social and Preventive Medicine (ISPM) University of Bern, Bern, Switzerland

\section{Correspondence to} Dr Yvonne Pfeiffer, Patient Safety Foundation, Zurich, ZH 8032, Switzerland;

Pfeiffer@patientensicherheit.ch

Accepted 24 January 2020 Published Online First 18 February 2020

\section{SLinked}

- http://dx.doi.org/10.1136/ bmjqs-2019-009552

\section{Check for updates}

(C) Author(s) (or their employer(s)) 2020. No commercial re-use. See rights and permissions. Published by BMJ.

To cite: Pfeiffer $Y$ Zimmermann C, Schwappach DLB. BMJ Qual Saf 2020;29:536-540.
Double checking is often considered a useful strategy to detect and prevent medication errors, especially before the administration of high-risk drugs. ${ }^{12}$ From a safety research perspective, the effectiveness of double checking in preventing medication errors is limited by several factors, ${ }^{34}$ even if they are conducted independently ${ }^{5}$ : a double check represents a barrier designed to catch errors before they reach the patient. If it is carried out by two people (compared with a technology-based check, like barcode scanning), the detection rate is limited because both people may be affected by the same disturbances in the environment, for example, noise, confusing drug labels or cognitive biases in information processing (eg, confirmation bias ${ }^{6}$ ). Double checks also may become a mindless routine over time, ${ }^{37}$ meaning that the checking persons rely on the other check and are not as attentive as they could be. In addition, checking persons may not dare to raise an identified error to a person of higher authority status. ${ }^{8}$

As double checking uses considerable resources of nurses' time and cognitive capacity, ${ }^{9}$ there is a pressing need to know whether existing empirical evidence supports using double checking despite its mentioned shortcomings. In this issue, Koyama et $a l^{9}$ helped address this gap by reviewing empirical research on the effectiveness of double checking as a patient safety intervention. Just like Alsulami et al in $2012,{ }^{10}$ they come 7 years later to the same conclusion: double checking lacks sound empirical evidence. Out of the 13 studies included in the review, there are only three good-quality studies, ${ }^{11-13}$ one of which provided evidence for double checks reducing medication error. ${ }^{13}$ Most studies lacked methodological rigour, for example, in applying insufficient methods for assessing the outcomes. No study investigated the relation between double checking and medication-related patient harm, and most studies did not assess adherence with double-checking procedures. An important point raised in the review was that very few studies defined the specific actions (eg, which items to check or the kind of procedure used) required in the double check-in other words, what 'double-checking' meant. Only three studies specified whether they studied independent double checking. In addition, only 2 out of 13 studies reported the work steps in the medication process requiring double checking.

The conclusion that the empirical evidence on such a resource-intensive and widespread practice is scarce is sobering. In alignment with Hewitt et $a l,{ }^{4}$ we propose to work on the missing clarity of the concept of double check in order to be able to generate more substantial evidence in future.

First, specific descriptions for different double checking procedures need to be developed. Currently, various checking procedures are covered under the umbrella term double checking ${ }^{14-16}$ : for example, one nurse checking two times a prepared drug against the prescription, two nurses performing two checks sequentially or together, for example, one nurse reading aloud the prescription while the other nurse listens and checks the label and then in a second step reads back the label to the other nurse who checks against the prescription (read-read back procedure ${ }^{15}$ ). Another example for the missing clarity of the concept of double checking is that double checks have often been defined as requiring two persons, ${ }^{11}$ while single-person double-checking has also been proposed as a checking strategy. ${ }^{16}$ In order to systematise the various kinds of checking procedures, we developed the framework presented in the following. Based on this differentiation, any future review should analyse and report the 
'type of double check' to foster comparability and ease of interpretation of the results.

Second, it is important to draw a line between checking and activities that are covered by the term today, but require very different cognitive activities. As White et $a l^{17}$ pointed out, double checking a set of prepared drugs against the prescription is a rather mechanistic activity, demanding a person's attention, but not their critical thinking. Currently, activities requiring critical thinking are often called double checking, too, for example, (1) determining whether a dose calculation is correct ${ }^{14}$ and (2) identifying an error in the prescription, such as the weight-based errors in the simulation study by Douglass et al. ${ }^{13}$

Guided by our own research, we present a framework for classifying checking procedures and differentiating them from other medication-related safety behaviours in order to structure future research and practice. In addition, the concept of independence is discussed.

\section{FRAMEWORK FOR DEFINING CHECKING BEHAVIOURS}

We propose the following definition of checking: A check is a comparison of information stemming from two (or more) different sources (eg, prescription vs label of an IV-bag of chemotherapy). For a double check, the same comparison is performed twice. Thus, it is not the number of persons or points in time but the number of comparisons between information sets that is the criterion to distinguish a double from a single check. Important to note is that a check may also be performed by a machine, for example, in comparing the drug and the prescription using barcode scanning.

Depending on how many times an information comparison is conducted and how many persons are involved in the check, different kinds of checks can be differentiated (see table 1 and online supplementary figure 1). The most common checking procedures are single checks, and double checks by two persons, which may either be performed sequentially after each other or simultaneously in a common read-read back procedure. Table 1 shows how different ways to involve persons in a double check can be systematically differentiated. Many of the possibilities are not used in daily practice. It is theoretically possible that four persons conduct a double check, for example, two different pairs of persons perform a read-read back procedure (see table 1 ).
Differentiating plausibility reviews from checking

Building on White et al's proposition to differentiate checking and critical thinking as requiring different cognitive modes, we define critical thinking, the use of a professional's own knowledge, as a plausibility review. In a plausibility review, information is not compared, but evaluated: for example, when a nurse checks a prescription and realises that the drug needs to be diluted in a different carrier solution. The nurse identifies the error by using own knowledge. Plausibility reviews are common in healthcare, at least implicitly, and are often executed without being part of standard protocols or written-down procedures. ${ }^{18}$

\section{Differentiating information generation from checking} In particular in high-risk environments like intensive and cancer care, nurses often need to calculate flow rates or dosages or determine them from a table. Calculations are often seen as a part of a double check, ${ }^{15}$ particularly when a second person is involved. ${ }^{19}$ In our framework, we consider them as generating (in contrast to comparing) information. The calculated or determined value is new, 'generated', information. If subsequently the two calculated values were compared with each other, this activity would be considered a (single) check according to our framework (see online supplementary figure 1). Table 2 shows four important questions to be asked in order to be able to determine whether a certain medication safety-related activity is a check, a calculation or a plausibility review.

\section{Clarifying the concept of independence}

Independence in double checking is frequently recommended, ${ }^{19}$ but the concept has been not very well adopted or understood in practice, ${ }^{15} 20$ and rarely differentiated in research. ${ }^{9}$ We suppose that useful recommendations of how to design independent checks are lacking because of the missing clarity of the concept of checks. The usual example brought up to describe an independent check is a calculation, that is, instead of telling someone to check if a certain number of pills is correct, one should ask the other person to count the pills again. ${ }^{19}$ Technically, from our framework's perspective, the concept of independence is applied to information generation in this example and not to information comparison. An independent calculation means that a clinician uses no prior

Table 1 Kinds of checking procedures

\begin{tabular}{lll}
\hline & \multicolumn{2}{c}{ First check performed by } \\
\cline { 2 - 3 } Second check performed by & One person & A pair of persons \\
\hline The same person & Double check by a single person & Double check by a pair of persons and one person \\
Another person & Double check by two single persons & Double check by a pair of persons and one single person \\
The same pair & Double check by one person and one pair of persons & Double check by a pair of persons \\
Different pair & $\begin{array}{l}\text { Double check by one single person and a pair of } \\
\text { persons }\end{array}$ & Double check by two pairs of persons \\
\hline
\end{tabular}


Table 2 Differentiating plausibility reviews, calculations and checks

\begin{tabular}{|c|c|c|c|c|}
\hline \multirow[b]{2}{*}{ Questions to ask } & \multicolumn{4}{|c|}{ Kind of activity } \\
\hline & Single check & Double check & Calculation & Plausibility review \\
\hline Are two sources of information being compared? & Yes & Yes & & \\
\hline Are two sources of information being compared twice? & & Yes & & \\
\hline Is information being generated (eg, doses)? & & & Yes & \\
\hline $\begin{array}{l}\text { Is own knowledge being used to evaluate information (eg, reviewing a } \\
\text { prescription)? }\end{array}$ & & & & Yes \\
\hline
\end{tabular}

information in order to avoid confirmation bias. One way to reduce confirmation bias is to have the second person generate the information (eg, count the pills) before looking at the information to be compared (eg, the pill count provided by the first person). That is, the second person must (1) count the pills without prior knowledge of the first person's count, (2) document the information (ie, the generated pill count) and (3) compare the two sources of information (ie, first person's count and the second person's count). Thus, regarding the calculation of a dose for example, the concept of independence works well to differentiate procedures, that is, independent versus 'do and show, together, and watching ${ }^{14}$ procedures, which do not control for confirmation bias. However, what does independence mean if it is applied to information comparison, that is, checking? Priming is much harder to avoid for checking than for calculating or counting because in order to compare information, one always needs to read it first, which basically is a form of priming. Therefore, full independence cannot be achieved for checks and optimising independence works differently: in order to reduce confirmation bias, ${ }^{6}$ it is essential to design procedures that actualise as little prior knowledge about the information to check as possible and to reduce contextual influence. Reading numbers from right to left in comparing a programmed infusion rate to the prescription may minimise for example the influence of confirmation bias. For performing good checks, the automatic cognitive efforts of sense-making need to be reduced as much as possible. In contrast, for plausibility reviews, one's own knowledge and sense-making need to be deliberately actualised. We therefore propose to differentiate between calculations, plausibility reviews and checks in order to make useful recommendations on independence. Thus, for calculations, the traditional concept of independence can be applied and confirmation bias can be avoided in designing good procedures, while for checks the influence of confirmation bias only can be limited; for plausibility reviews, independence is not relevant, as confirmation bias is not the important issue, it rather should be designed so that the capacity of an individual to actualise own knowledge is maximised (ie, not being interrupted, a calm environment, a dedicated space to perform the review). In addition to Koyama et al's work, we propose to stop using the concept of primed checks, and instead to describe the ways in which checking procedures are designed to reduce the influence of confirmation bias.

\section{WHAT DOES THE FRAMEWORK ADD TO RESEARCH?}

In their review, Koyama et al provide important information on outcome measures, outcome measure assessment methods and study designs. However, they do not differentiate double-checking methodsmerely because this information is often not sufficiently provided in original studies. We believe that without a clear definition of 'checking procedures' the evidence base for double-checking will remain at best vague-simply because it is unclear what the investigated intervention precisely is. We presented a framework to conceptualise the various activities covered under the term double check along the informationprocessing tasks they consist of. Evaluating the correctness of a prescription may best be done in performing a plausibility review, while checking whether one is about to administer it to the right person or whether a programmed infusion rate is correct represents a typical task to be fulfilled in performing a (double) check.

Currently, nursing guidelines (eg, Neuss et $a l^{21}$ ) and hospital nursing procedures are not describing the specific procedure to be performed in a double check. $^{22}$ In using precise concepts, guidelines may better support clinical practice. For example, reflective thinking activities are usually not described in standards and protocols, despite being potentially very effective in catching errors. ${ }^{18}$ Interestingly, White et al reported that integrating a question designed to trigger critical thinking in a checklist of a checking procedure did not improve the identification of clinical decision errors in their study. The authors concluded that the 'mechanistic ${ }^{17}$ cognitive mode of information processing that is necessary during a check may not translate well into a more reflective thought process. It seems likely that humans have difficulties in switching between these two modes immediately. Thus, distinguishing plausibility reviews from checking is very important to design adequate medication safety processes, for example, in defining different points in time or locations. Creating space and points in time in the medication process for plausibility reviews represents a powerful avenue 
to institutionalise reflective thinking ${ }^{18}$ as a means to catch errors.

Our conceptualisation allows for interpreting prior results from a new perspective, for example, the simulation study by Douglass et $a l^{13}$ that was positively evaluated in the review. ${ }^{9}$ The two errors planted in the simulation required two different kinds of cognitive activity to be detected: while the wrong vial could be identified in comparing information (performing a check), the identification of the wrong dose required the use of own knowledge, thus a plausibility review. As reported above, plausibility reviews may not be effective if conducted within a checking situation because it needs critical thinking instead of mechanistic information comparison. The fact that the wrong dose error actually needed critical thinking to be identified may be the main explanation for the finding that less errors were identified in the wrong dose scenario, in addition to the reason that different drugs were used for the single and the double check scenarios. ${ }^{23}$

Although the framework presents various forms of double checks, more specifications are necessary for describing how an actual double check should be performed: the items to check, the position of the check within the process as well as the steps of the actual check need to be specified; if more than one person are involved in the checking procedure, the way the involved persons collaborate needs to be specified, too. Similarly, for calculations, it needs to be specified by whom dosages of high-risk drugs need to be calculated, whether this needs to be performed twice and by whom, how, and how often the result needs to be checked.

\section{POTENTIAL FUTURE USE OF THE FRAMEWORK}

The presented framework conceptualising double checking is intended to serve research and practice. In providing a basis for specifying the activity investigated, future effectiveness studies will be easier to plan, compare and evaluate in their significance. We hope that in using the specific descriptions of checking procedures, future studies will more easily build on each other. Translating empirical evidence into practice will also be easier if the specific procedures studied are known and described. Furthermore, guidelines and standard operating procedures will hopefully benefit from a more concise use of concepts. The framework's concepts furthermore are useful to assess the types of checks performed along a medication process by different professional groups to identify loopholes and redundancies. $^{22}$

Contributors All authors contributed to the development of the framework. YP wrote the manuscript which was revised by DS.

Funding The framework was developed within two projects that were supported by a research grant from Krebsforschung Schweiz (Cancer Research Switzerland, grant No. KFS-349608-2014), by an unrestricted research grant from the HanelaStiftung, as well by a grant from the National Office of Public
Health (grant \#500.0005-1/8). The funding sources had no influence on the development of the framework.

Competing interests None declared.

Patient consent for publication Not required.

Provenance and peer review Not commissioned; externally peer reviewed.

\section{ORCID iDs}

Yvonne Pfeiffer http://orcid.org/0000-0001-6226-5037

David L B Schwappach http://orcid.org/0000-0001-8668-3065

\section{REFERENCES}

1 Subramanyam R, Mahmoud M, Buck D, et al. Infusion medication error reduction by two-person verification: a quality improvement initiative. Pediatrics 2016;138:e20154413-11.

2 Schwappach DLB, Taxis K, Pfeiffer Y. Oncology nurses' beliefs and attitudes towards the double-check of chemotherapy medications: a cross-sectional survey study. BMC Health Serv Res 2018;18:123.

3 ArmitageGA. Affiliation: the risks of double checking. Nurs Manag 2009;16:30-5.

4 Hewitt T, Chreim S, Forster A. Double checking: a second look. J Eval Clin Pract 2015:1-8.

5 U D. Medication Safety Alerts. Double checking: does it work? Can J Hosp Pharm 2003;56:167-9.

6 Nickerson RS. Confirmation bias: a ubiquitous phenomenon in many Guises. Rev Gen Psychol 1998;2:175-220.

7 Tamuz M, Harrison MI. Improving patient safety in hospitals: contributions of high-reliability theory and normal accident theory. Health Serv Res 2006;41:1654-76.

8 Armitage G. Double checking medicines: defence against error or contributory factor? J Eval Clin Pract 2008;14:513-9.

9 Koyama AK, Maddox C-SS, Li L, et al. Effectiveness of double checking to reduce medication administration errors: a systematic review. BMJ Qual Saf 2020;29:595-603.

10 Alsulami Z, Conroy S, Choonara I. Double checking the administration of medicines: what is the evidence? A systematic review. Arch Dis Child 2012;97:833-7.

11 Alsulami Z, Choonara I, Conroy S. Paediatric nurses' adherence to the double-checking process during medication administration in a children's hospital: an observational study. $J$ Adv Nurs 2014;70:1404-13.

12 Härkänen M, Ahonen J, Kervinen M, et al. The factors associated with medication errors in adult medical and surgical inpatients: a direct observation approach with medication record reviews. Scand J Caring Sci 2015;29:297-306.

13 Douglass AM, Elder J, Watson R, et al. A randomized controlled trial on the effect of a double check on the detection of medication errors. Ann Emerg Med 2017:1-10.

14 Dickinson A, McCall E, Twomey B, et al. Paediatric nurses' understanding of the process and procedure of doublechecking medications. J Clin Nurs 2010;19:728-35.

15 Schwappach DLB, Pfeiffer Y, Taxis K. Medication doublechecking procedures in clinical practice: a cross-sectional survey of oncology nurses' experiences. BMJ Open 2016;6:e011394-10.

16 Feng X, Zhu L, Zhou Q. The checking methods before medication administration: a perspective from a Joint Commission International-accredited academic medical center hospital in China. J Eval Clin Pract 2016:1-3.

17 White RE, Trbovich PL, Easty AC, et al. Checking it twice: an evaluation of checklists for detecting medication errors 


\section{Editorial}

at the bedside using a chemotherapy model. BMJ Qual Saf 2010;19:562-7.

18 Rohde E, Domm E. Nurses' clinical reasoning practices that support safe medication administration: an integrative review of the literature. J Clin Nurs 2018;27. doi:10.1111/jocn.14077

19 Institute for Safe Medication Practices. Medication Safety Alert! Independent double checks: undervalued and misused. Selective use of this strategy can play an important role in medication safety. ISMP Medicat. Saf. Alert 2013;18:1-4.

20 Institute for safe medication practices (Canada). Independent double checks-are your checks truly independent? ISMP Canada SAF. Bull 2019;19:4.
21 Neuss MN, Gilmore TR, Belderson KM, et al. Updated American Society of Clinical Oncology/Oncology Nursing Society chemotherapy administration safety standards. Including Standards for Pediatric Oncology 2016;2017:12.

22 Pfeiffer Y, Gut SS, Schwappach DLB. Medication safety in oncology care: mapping checking procedures from prescription to administration of chemotherapy. J Oncol Pract 2017;026427.

23 Berdot S, Sabatier B. Medication errors may be reduced by double-checking method. Evid Based Nurs 2018;0:2018. 